\title{
Semi-obnoxious location models: A global optimization approach
}

\author{
Dolores Romero-Morales \\ Rotterdam School of Management, Erasmus University Rotterdam. \\ P.O. Box 1738, 3000 DR Rotterdam, The Netherlands \\ Emilio Carrizosa \\ Eduardo Conde \\ Facultad de Matemáticas, Universidad de Sevilla. \\ C/ Tarfia, s/n 41012 Sevilla, Spain
}

$21 / 07 / 1997$

\begin{abstract}
In the last decades there has been an increasing interest in environmental topics. This interest has been reflected in modeling the location of obnoxious facilities, as shown by the important number of papers published in this field. However, a very common drawback of the existing literature is that, as soon as environmental aspects are taken into account, economical considerations (e.g. transportation costs) are ignored, leading to models with dubious practical interest. In this paper we take into account both the environmental impact and the transportation costs caused by the location of an obnoxious facility, and propose as solution method of the well-known BSSS, with a new bounding scheme which exploits the structure of the problem.
\end{abstract}

Keywords: Location Theory, Global Optimization, Semi-obnoxious facilities, Big-squares Small-squares

\section{Introduction}

In the last decades there has been an increasing interest for environmental problems, mainly due to industrial and technological innovations (most causing environmental deterioration), and recent ecological disasters.

This interest has been reflected in the literature of Locational Analysis, as shown by the significant number of papers devoted to modeling the location of 
undesirable facilities, $[1,6,18,19]$, such as nuclear or chemical plants, garbage dumps, plants for treatment of residual waters, ....

These facilities decrease the welfare of neighboring population centers, thus remoteness is seen by population as a positive attribute, in contrast with the classical location models, where the facility is desired as close as possible. However, together with the environmental impact, the location of the facility carries out a usually important economical cost, due to transportation costs.

Although most models in the literature addressing the location of these facilities simply ignore transportation costs, [7], which is rather extremely unrealistic, some papers accommodate both aspects.

For instance, in $[4,16]$ both transportation costs and environmental impact (considered also as a cost) are assumed to be linear functions of the distances between the facility and the population centers. In order to obtain more realistic models, in the review [6] the environmental cost is suggested to be modeled as a nonincreasing convex function of the distances, whilst the transportation cost can be better modeled as Lipschitz-continuous nondecreasing function of distances, [8].

In this paper we follow these suggestions and propose to locate the facility at the optimal solution of the mathematical programme

$$
\inf _{x \in S} z(x)=\sum_{a \in A}\left[f_{a}\left(\|x-a\|_{-}^{a}\right)+g_{a}\left(\|x-a\|_{+}^{a}\right)\right]
$$

where

- $A \subset \mathbb{R}^{2}$ is a nonempty finite set, representing the coordinates of the population centers.

- $S \subset \mathbb{R}^{2}$ is a nonempty closed (possibly unbounded) set, given by a finite union of polygonal regions, and representing the set of feasible locations for the facility. We make the standard assumption in Continuous Location that $S \backslash A \neq \emptyset$.

- $\|\cdot\|_{-}^{a},\|\cdot\|_{+}^{a}$ are arbitrary gauges in $\mathbb{R}^{2}$, associated with the population center $a$. For more see $[5,18,20,22]$.

- $f_{a}:(0,+\infty) \longmapsto \mathbb{R}$ is a nonincreasing convex function measuring the environmental impact (cost) on the population center $a$ caused by a facility at a distance $\|x-a\|_{-}^{a}$ from $a$.

Function $f_{a}$ is extended to $[0,+\infty)$, defining it at 0 as

$$
f_{a}(0)=\lim _{t \downarrow 0} f_{a}(t) \in \mathbb{R} \cup\{+\infty\} .
$$


- $g_{a}:[0,+\infty) \longmapsto \mathbb{R}$ is a nondecreasing Lipschitz-continuous function, measuring the transportation costs from the population center $a$ to a facility at a distance $\|x-a\|_{+}^{a}$.

It should be mentioned that particular instances of (1) have been previously addressed in the literature, $[10,18]$, mainly considering the functions $f_{a}$ and $g_{a}$ as linear functions, using particular cases of gauges (norms), and not considering mixed gauges but assuming instead that $\|\cdot\|_{-}^{a}=\|\cdot\|_{+}^{a}=\|\cdot\|$, for all $a \in A$.

For instance, in [3] the feasible region is assumed to be given as a finite union of polytopes and the gauge in use for both factors and any population center is the euclidean norm; the functions are assumed to be linear, and the problem is solved by general-purpose techniques developed for d.c. functions, i.e., functions which can be written as the difference of two convex functions, [13]. They also consider a nonlinear case, in which $f_{a}(t)=\omega_{a} e^{-t}$, with $\omega_{a}>0$, leading again to a d.c. optimization problem, i.e., a problem with d.c. objective function and constraints given as level sets of d.c. functions.

In [22] a particular instance of (1) is addressed, in which the feasible region is a convex polygon, and each $f_{a}$ and $-g_{a}$ are nonincreasing convex functions defined on $\mathbb{R}_{+}$. The authors show that, under some assumptions, the objective function is d.c., and solvable by d.c. optimization techniques, [13].

However the most versatile technique proposed so far for semiobnoxious facilities-location problems is a continuous branch-and-bound method, BSSS, proposed in [7] to solve a particular instance of (1), namely, the case that, for each $a \in A$, $f_{a}$ is a nonincreasing continuous function and $g_{a}(t)=0$, for all $t \in[0,+\infty)$. See also Section 3.

The remainder of the paper is structured as follows. In Section 2 we address the problem of existence of an optimal solution for (1), and present some sufficient conditions. Under such conditions, we propose in section 3 as solution technique the BSSS algorithm developed in $[7,8]$ in order to exploit better the structure of (1). In section 4 some preliminary computational results are presented.

\section{Existence of an optimal solution}

The model proposed enables the functions $f_{a}$ tending to $+\infty$ when distances tend to zero, i.e., when the facility is located near a population center. We first show that such locations are nonoptimal.

It is well-known that any two gauges $\|\cdot\|_{1},\|\cdot\|_{2}$ are equivalent, i.e., there exist constants $C_{1}>0, C_{2}>0$ such that

$$
C_{1}\|x\|_{1} \leq\|x\|_{2} \leq C_{2}\|x\|_{1} \quad \forall x \in \mathbb{R}^{2} .
$$


Hence, there exist strictly positive constants $C_{+}, C_{-}$and $K_{1}, K_{2}$ such that for any $x \in \mathbb{R}^{2}, a, b \in A$

$$
\begin{aligned}
\|x\|_{+}^{a} & \leq C_{+}\|x\|_{+}^{b} \\
\|x\|_{-}^{a} & \leq C_{-}\|x\|_{-}^{b} \\
K_{1}\|x\|_{-}^{a} & \leq\|x\|_{+}^{a} \\
K_{2}\|x\|_{+}^{a} & \leq\|x\|_{-}^{a} .
\end{aligned}
$$

Let

$$
A_{\infty}=\left\{b \in A: \lim _{t \downarrow 0} f_{b}(t)=+\infty\right\} .
$$

Theorem 1 There exists $H=\bigcup_{b \in A_{\infty}} B_{-}^{b}, B_{-}^{b}$ being an open neighborhood of $b$, for all $b \in A_{\infty}$, pairwise disjoint, such that

$$
\inf _{x \in S} z(x)=\inf _{x \in S \backslash H} z(x) .
$$

The proof can be found in the Appendix.

Remark 1 As can be seen in the proof of Theorem 1, without loss of generality, one can assume that the sets deleted around each point in $A_{\infty}$ are squares, by inscribing a square within each $B_{-}^{b}$. With this, the resulting set $S \backslash H$ can be written as a finite union of polygonal regions, thus inheriting the geometrical structure of $S$.

This is illustrated in Figure 1. In this picture we have

$$
\begin{aligned}
A & =\left\{a_{1}, a_{2}, a_{3}, a_{4}, a_{5}, a_{6}, b_{1}, b_{2}, b_{3}\right\} \\
A_{\infty} & =\left\{b_{1}, b_{2}, b_{3}\right\} .
\end{aligned}
$$

Around each point in $A_{\infty}$ we have drawn the corresponding neighborhood, using the gauge $\|\cdot\|_{-}^{b_{i}}$, with radius equal to $t_{0}\left(b_{i}\right)$, for $i=1,2,3$. Inside each neighborhood we have inscribed a square $C_{i}$, for $i=1,2,3$. Then we can assume the new feasible region, containing an optimal solution of (1), to be $S \backslash\left(\bigcup_{i=1}^{3} C_{i}\right)$.

In what follows we give some sufficient conditions under which Problem (1) attains its optimal value, i.e., it has an optimal solution.

First, if $S$ is bounded, by Theorem 1, solving (1) amounts to minimizing a continuous (and finite!) function on a compact set. Hence one has the following

Theorem 2 If the feasible region $S$ is bounded, then Problem (1) has an optimal solution. 


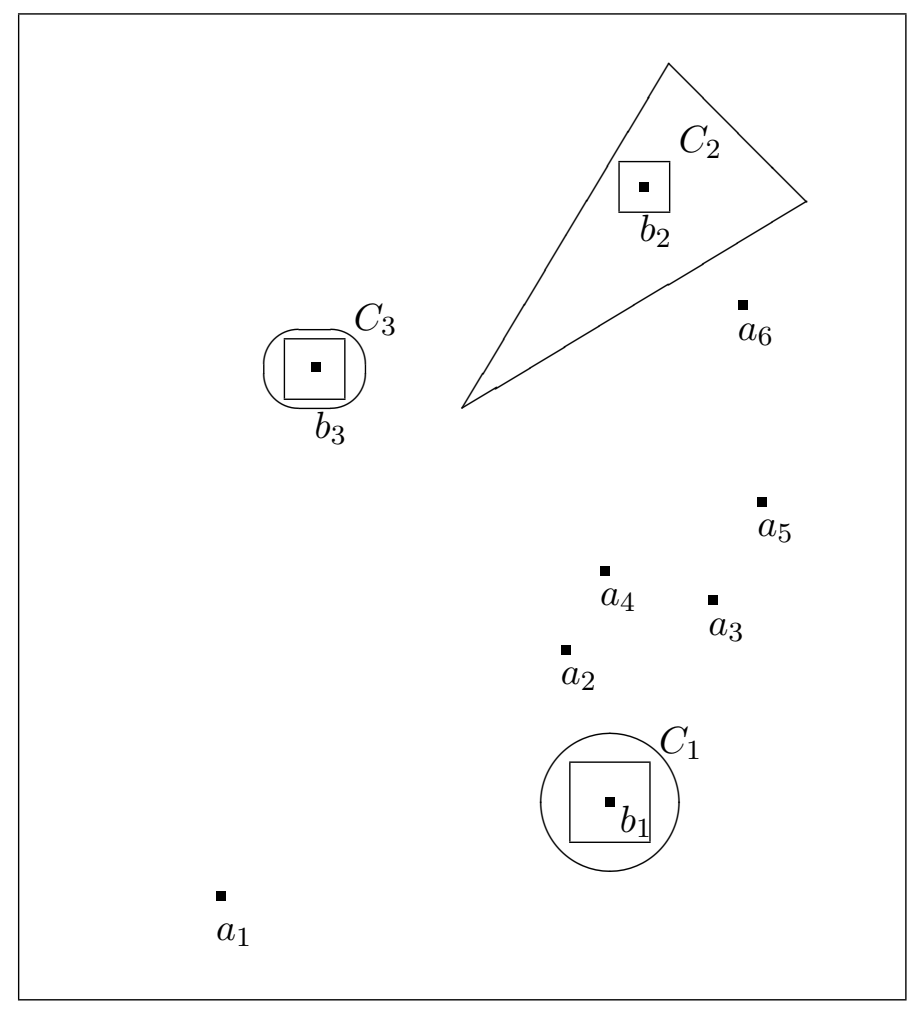

Figure 1: Erasing squares around points in $A_{\infty}$

Although in practice the feasible region will always be bounded, the study of problems with unbounded domain (e.g. $S=\mathbb{R}^{2}$ ) may also be of interest. The sufficient condition, under which an optimal solution of (1) exists, obtained in [4] for the unconstrained case is generalized in [2], supposing transportation cost measured as concave and nondecreasing function of the distance. Roughly speaking, such condition states that, if transportation costs are big enough (compared with the environmental impacts) for points far away, the optimal value is attained:

Theorem 3 [2] Let $C_{-}$and $K_{1}$ be positive constants verifying respectively (4) and (5). If function $g_{a}$ is concave, for all $a \in A$, and

$$
\lim _{t \uparrow+\infty} \sum_{a \in A}\left[f_{a}\left(C_{-} t\right)+g_{a}\left(K_{1} t\right)\right]=+\infty,
$$

then Problem (1) has an optimal solution.

Remark 2 As can be seen in [2], under the assumptions of Theorem 3, one can always construct a rectangle containing the optimal solution of Problem (1), and then, as soon as the functions $g_{a}$ are concave, one can assume without loss of generality that the feasible region is bounded. 


\section{The BSSS with sharper bounds}

According to Remarks 1, 2, one can start with a feasible region consisting of a finite union of bounded polygonal regions, within which the objective function is continuous. In particular, hereafter we assume that Problem (1) has an optimal solution.

Since the objective function of Problem (1) is, in general, non-convex, if the global optimum is sought, Global-Optimization tools are required, [9, 13].

In this sense, the algorithm proposed here to solve Problem (1) is the wellknown BSSS (Big Square Small Square), with an improvement in its bounding process.

The BSSS method is a Branch-and-Bound procedure, proposed by Hansen et al., $[7,8]$, and modified by Plastria, [17], whose success is due to its simplicity and versatility to solve planar problems.

In this case, setting as the feasible region the set $S \backslash H$ given in Theorem 1 (or better, the region described in Remark 1), it starts with the construction of a rectangle $R_{0}$ known to contain an optimal solution for (1) (see Remark 2 ), which is dynamically divided into smaller rectangles, discarding from further considerations those checked to be infeasible or nonpromising (if a lower bound of $\min _{x \in R} z(x)$ is greater than the value of $z(\cdot)$ at some inspected point, $R$ cannot contain an optimal solution, thus can be ruled out).

We do not go into further details on the scheme of the algorithm, which is well documented e.g. in $[7,8,10,17]$ and focus our attention on our contribution to the basic BSSS, namely, how to obtain sharper lower bounds for $\min _{x \in R} z(x)$ for a given rectangle $R$.

\section{$3.1 \quad$ Bounding}

In what follows, $R$ denotes a rectangle and $z^{*}(R)$ is the optimal value of $z(x)$ on $R$, namely

$$
z^{*}(R)=\min _{x \in R} z(x)
$$

In this subsection we propose a lower bound for $z^{*}(R)$, using Lagrangean relaxation techniques, [15], and compare the sharpness of this bound with respect to the bound proposed in $[7,8,17]$. By using the monotonicity of the functions $f_{a}$, $g_{a}$, one obtains the following crude bound.

Proposition 1 Let $z_{0}(R)$ be given by

$$
z_{0}(R)=\sum_{a \in A}\left[f_{a}\left(\max _{x \in R}\|x-a\|_{-}^{a}\right)+g_{a}\left(\min _{x \in R}\|x-a\|_{+}^{a}\right)\right] .
$$

Then, $z_{0}(R) \leq z^{*}(R)$. 
Remark 3 The bound $z_{0}(R)$ is easily computed after computing the highest distance (following $\|\cdot\|_{-}^{a}$ ) from each $a \in A$ to the rectangle $R$, and the projection (following $\|\cdot\|_{+}^{a}$ ) of such points onto $R$.

Since the function $\|\cdot-a\|_{-}^{a}$ is convex, it attains its maximum on $R$ at some vertex of $R$. Hence finding $\max _{x \in R}\|x-a\|_{-}^{a}$ reduces to evaluating the four vertices of $R$.

On the other hand, although calculating $\min _{x \in R}\|x-a\|_{+}^{a}$ amounts in general to solve a convex programme, [12], a straightforward procedure has been suggested for the case in which $\|\cdot\|_{+}^{a}$ is an $l_{p}$ norm, see $[8,17]$ for details.

We now obtain a different lower bound for $z^{*}(R)$ and show that it is at least as sharp as $z_{0}(R)$.

Since

$$
\begin{aligned}
z^{*}(R) & \geq \min _{x \in R} \sum_{a \in A} f_{a}\left(\|x-a\|_{-}^{a}\right)+\min _{x \in R} \sum_{a \in A} g_{a}\left(\|x-a\|_{+}^{a}\right) \\
& \geq \min _{x \in R} \sum_{a \in A} f_{a}\left(\|x-a\|_{-}^{a}\right)+\sum_{a \in A} g_{a}\left(\min _{x \in R}\|x-a\|_{+}^{a}\right) \\
& \geq z_{0}(R)
\end{aligned}
$$

it follows that a sharper lower bound for $z^{*}(R)$ can be obtained by bounding

$$
\min _{x \in R} \sum_{a \in A} f_{a}\left(\|x-a\|_{-}^{a}\right) .
$$

The following proposition provides an equivalent formulation of (11), which allows us use Lagrangean relaxation techniques, [15], in order to obtain a lower bound of (11).

Proposition 2 For each $a \in A$, let $I_{a}$ be an interval of $\mathbb{R}_{+}$containing

$$
\left[\min _{x \in R}\|x-a\|_{-}^{a}, \max _{x \in R}\|x-a\|_{-}^{a}\right] .
$$

Then, Problem (11) is equivalent to

$$
\begin{aligned}
\min & \sum_{a \in A} f_{a}\left(t_{a}\right) \\
& x \in R, \\
& t_{a} \in I_{a} \quad \forall a \in A \\
& t_{a} \leq\|x-a\|_{-}^{a} \quad \forall a \in A
\end{aligned}
$$




\section{Proof.}

- Let $x^{*}$ be optimal for Problem (11). Defining $t^{*}=\left(\left\|x^{*}-a\right\|_{-}^{a}\right)_{a \in A}$, it follows that $\left(x^{*}, t^{*}\right)$ is optimal for $(12)$, since any other feasible solution $(\hat{x}, \hat{t})$ for (12) verifies

$$
\sum_{a \in A} f_{a}\left(t_{a}^{*}\right)=\sum_{a \in A} f_{a}\left(\left\|x^{*}-a\right\|_{-}^{a}\right) \leq \sum_{a \in A} f_{a}\left(\|\hat{x}-a\|_{-}^{a}\right) \leq \sum_{a \in A} f_{a}\left(\hat{t}_{a}\right) .
$$

- Let $\left(x^{*}, t^{*}\right)$ be optimal for (12). Then $x^{*}$ is optimal for (11), since for any $\hat{x}$ feasible for (11), defining $\hat{t}=\left(\|\hat{x}-a\|_{-}^{a}\right)_{a \in A}$, one obtains

$$
\sum_{a \in A} f_{a}\left(\left\|x^{*}-a\right\|_{-}^{a}\right) \leq \sum_{a \in A} f_{a}\left(t_{a}^{*}\right) \leq \sum_{a \in A} f_{a}\left(\hat{t}_{a}\right)=\sum_{a \in A} f_{a}\left(\|\hat{x}-a\|_{-}^{a}\right) .
$$

Using the Lagrangean relaxation, [15], of the constraints $t_{a} \leq\|x-a\|_{-}^{a}$ one then obtains:

Proposition 3 For each $\lambda=\left(\lambda_{a}\right)_{a \in A} \geq 0$, the value $R L_{-}(\lambda)$ defined as

$$
\begin{aligned}
R L_{-}(\lambda) & =\min _{\substack{x \in R \\
t_{a} \in I_{a}}} \sum_{a \in A}\left[f_{a}\left(t_{a}\right)+\lambda_{a}\left(t_{a}-\|x-a\|_{-}^{a}\right)\right] \\
& =\left(\sum_{a \in A} \min _{t_{a} \in I_{a}}\left[f_{a}\left(t_{a}\right)+\lambda_{a} t_{a}\right]\right)-\max _{x \in R} \sum_{a \in A} \lambda_{a}\|x-a\|_{-}^{a}
\end{aligned}
$$

is a lower bound of the value (11).

Remark 4 Evaluating $R L_{-}(\lambda)$ amounts to finding, for each $a \in A$, the optimum of a univariate convex optimization problem, for which, in some important cases, closed formulae can be easily derived. See [21].

Remark 5 The bound $R L_{-}(\lambda)$ can be sharpened by considering all the possible vectors $\lambda \geq 0$. In this case one needs to solve a nondifferentiable concave maximization problem, which requires Convex Optimization techniques, [12]. As an alternative, one may get in practice an approximation by performing a few iterations of e.g. the subgradient method, [11], see also Subsection 3.2.

By setting, for each $a \in A, I_{a}=\left[0, \max _{x \in R}\|x-a\|_{-}^{a}\right]$, one gets

$$
\max _{\lambda \geq 0} R L_{-}(\lambda) \geq R L_{-}(0)=\sum_{a \in A} f_{a}\left(\max _{x \in R}\|x-a\|_{-}^{a}\right) .
$$

This yields the following relation between the bounds: 
Proposition 4 One has $z_{0}(R) \leq \max _{\lambda \geq 0} L(\lambda)$, where $L(\lambda)$ is defined by

$$
L(\lambda)=R L_{-}(\lambda)+\sum_{a \in A} g_{a}\left(\min _{x \in R}\|x-a\|_{+}^{a}\right) .
$$

\subsection{Convergence}

In order to solve Problem (1) one can use as lower bounds any of those proposed above.

Since $z(\cdot)$ is a Lipschitz function on $S \backslash H$, [20], and our proposed lower bound is at least as sharp as the one given in [17], see Proposition 4, we obtain a similar convergence result than in the case of BSSS with crude bound, see [17].

Theorem 4 Let $\varepsilon$ be the tolerance of the algorithm. After a finite number of iterations, the algorithm obtains an $\varepsilon$-optimal solution of Problem (1).

\section{Some numerical results}

Since the convergence of the procedure is obtained even for the crude bounds given by $L(0)$, it is necessary to check whether, in practice, the improvement of the bound deserves the computational effort required to optimize $L$.

For this reason we have performed a series of experiments, which, although do not provide a last word, are definitely encouraging.

For simplicity we generated a series of problems following the simulation scheme of $[3]$ :

$A$ is split into $J_{1}, J_{2}$, with

$$
f_{a}(t)=\left\{\begin{array}{ll}
0, & \text { if } \quad a \in J_{1} \\
\omega_{a} e^{-t}, & \text { if } \quad a \in J_{2}
\end{array} \quad g_{a}(t)=\left\{\begin{array}{lll}
\omega_{a} t, & \text { if } & a \in J_{1} \\
0, & \text { if } & a \in J_{2}
\end{array}\right.\right.
$$

The feasible region is the square with sides parallel to the coordinate axis, centered at point $(0.5,0.5)$ and of sidelength 1.1 ; weights are uniformly distributed in $[0,10]$. The gauge is always the euclidean norm and the accuracy is required is $\varepsilon=10^{-5}$.

We have implemented the BSSS algorithm with two bounding subroutines: one with crude bounds and a second one with lagrangean bounds; the optimal lagrangean multiplier is not calculated, but a fixed number (two) of iterations of the subgradient method, [11], were performed. The seed considered in this method is $R L_{-}(0)$ in order to improve the crude bound, see (13). 


\begin{tabular}{rrrrrr} 
& \multicolumn{3}{c}{$\begin{array}{c}\text { Lagrangean } \\
\text { bound }\end{array}$} & $\begin{array}{r}\text { Splitting } \\
\text { bound }\end{array}$ \\
\hline \hline$n$ & $p$ & $\mu_{\text {time }}$ & $\mu_{\text {iter }}$ & $\mu_{\text {time }}$ & $\mu_{\text {iter }}$ \\
\hline \hline & & & & & \\
100 & 10 & 8.0747402 & 1151.8 & 29.3716962 & 3180.3 \\
100 & 25 & 14.7353905 & 1455.3 & 286.7803445 & 13749.2 \\
200 & 25 & 13.7038284 & 979 & 45.9748721 & 3602.6 \\
200 & 50 & 32.1839499 & 1749.2 & 346.4267223 & 14410.2 \\
500 & 50 & 5.4511905 & 232 & 40.9912948 & 2233.2 \\
500 & 100 & 29.2207395 & 724.7 & 256.2111184 & 7994.4 \\
500 & 125 & 28.8807889 & 669.8 & 498.4821065 & 11328.3 \\
\hline
\end{tabular}

Table 1: Sharpness of lagrangean bound

Both procedures run in a Convex 240, ConvexOS V 10.1.197.1. for series of ten problems for different values of $|A|=n$ and $\left|J_{2}\right|=p$.

Table 1 shows, for different values of $n$ and $p$ the average CPU time $\left(\mu_{\text {time }}\right)$ and number of iterations $\left(\mu_{i t e r}\right)$ required.

It is evident that the Lagrangean bound requires much less computational effort than the crude bound. In passing, we observe that both CPU time and the number of iterations increase when the percentage of points in the set $J_{2}$ increases, in that case the splitting of the objective function into two independent parts damages the quality of the bound.

\section{Conclusions}

In this paper we have addressed the problem of locating a semiobnoxious facility in the plane, in which not only transportation costs, but also the environmental impact in population centers is important.

We show that this problem attains its optimal value, and the algorithm BSSS of Hansen et al. can be used to find it with a prespecified accuracy.

Finally we propose an alternative bounding procedure and present some comparative numerical results.

Although more intensive testing, (including polygonal highly nonconvex regions, for which other global optimization tools such as those in $[3,22]$ are not directly applicable) is needed, it seems evident from these very first numerical tests that the development of new bounding techniques for BSSS deserves being studied. 


\section{Appendix}

\section{Proof of Theorem 1.}

Let $x_{0} \in S \backslash A_{\infty}$ and $C_{-}>0$ verifying (4). By definition of the set $A_{\infty}$, one can take scalars $t_{0}(b) \in(0,1)$, for all $b \in A_{\infty}$ such that

$$
f_{b}(t)>z\left(x_{0}\right)-\sum_{\substack{a \in A \\ a \neq b}} f_{a}\left(C_{-}+\|b-a\|_{-}^{a}\right)-\sum_{a \in A} l_{a} \quad \forall t \in\left(0, t_{0}(b)\right) .
$$

Let

$$
\begin{aligned}
B_{-}^{b} & =\left\{x \in \mathbb{R}^{2}:\|x-b\|_{-}^{b}<t_{0}(b)\right\} \\
H & =\bigcup_{b \in A_{\infty}} B_{-}^{b}
\end{aligned}
$$

Without loss of generality, these neighborhoods may be assumed to be open and pairwise disjoint.

Taking $x \in S \cap H$, there exists exactly one $b \in A_{\infty}$ such that $\|x-b\|_{-}^{b}<t_{0}(b)$. For any other $a \in A, a \neq b$, it follows from the triangle inequality and the construction of $t_{0}(b)$ that

$$
\begin{aligned}
\|x-a\|_{-}^{a} & \leq\|x-b\|_{-}^{a}+\|b-a\|_{-}^{a} \\
& \leq C_{-}\|x-b\|_{-}^{b}+\|b-a\|_{-}^{a} \\
& <C_{-} t_{0}(b)+\|b-a\|_{-}^{a} \\
& <C_{-}+\|b-a\|_{-}^{a} .
\end{aligned}
$$

Hence,

$$
\begin{aligned}
& z(x)=\sum_{a \in A}\left[f_{a}\left(\|x-a\|_{-}^{a}\right)+g_{a}\left(\|x-a\|_{+}^{a}\right)\right] \\
& \geq \sum_{a \in A}\left[f_{a}\left(\|x-a\|_{-}^{a}\right)+l_{a}\right] \\
& =f_{b}\left(\|x-b\|_{-}^{b}\right)+\sum_{\substack{a \in A \\
a \neq b}} f_{a}\left(\|x-a\|_{-}^{a}\right)+\sum_{a \in A} l_{a} \\
& \geq f_{b}\left(\|x-b\|_{-}^{b}\right)+\sum_{\substack{a \in A \\
a \neq b}} f_{a}\left(C_{-}+\|b-a\|_{-}^{a}\right)+\sum_{a \in A} l_{a} \\
& >z\left(x_{0}\right)-\sum_{\substack{a \in A \\
a \neq b}} f_{a}\left(C_{-}+\|b-a\|_{-}^{a}\right)-\sum_{a \in A} l_{a}+\sum_{\substack{a \in A \\
a \neq b}} f_{a}\left(C_{-}+\|b-a\|_{-}^{a}\right)+\sum_{a \in A} l_{a} \\
& =z\left(x_{0}\right) .
\end{aligned}
$$


This implies both the non emptiness of $S \backslash H\left(x_{0} \in S \backslash H\right)$ and the domination of any point in $S \cap H$ by $x_{0}$, thus the result holds.

Acknowledgements. The authors thank some helpful comments from Manuel Muñoz Márquez, Juan José Salazar González and Alfredo Sánchez Navarro. The research of the second and third authors has been partially financed by the Dirección General de Investigación Científica y Técnica, Spain, under grant PB930927. This support is gratefully acknowledged.

\section{References}

[1] Bloemhof-Ruwaard, J.M., van Beek, P., HordiJk, L., van WassenHOVE, L.N., (1995), Interactions between Operational Research and Environmental Management, European Journal of Operational Research, 85, 229-243.

[2] Carrizosa, E., Romero-Morales D., (1995), Attainment of Optimal Solution in a Semiobnoxious Location Problem, Prepublicación de la Facultad de Matemáticas de la Universidad de Sevilla, Sección de Estadística e Investigación Operativa, $\mathbf{3 1 .}$

[3] Chen, P.C., Hansen, P., Jaumard, B., Tuy, H., (1992), Weber's Problem with Attraction and Repulsion, Journal of Regional Science, 32, 467-486.

[4] Drezner, Z., Wesolowsky, G.O., (1991), The Weber Problem on the Plane with some Negative Weights, INFOR, 29, 87-99.

[5] Durier, R., Michelot, C., (1985), Geometrical Properties of the FermatWeber Problem, European Journal of Operational Research, 20, 332-343.

[6] Erkut, E., Neuman, S., (1989), Analytical Models for Locating Undesirable Facilities, European Journal of Operational Research, 40, 275-291.

[7] Hansen, P., Peeters, D., Thisse, J.F., (1981), On the Location of an Obnoxious Facility, Sistemi Urbani, 3 299-317.

[8] Hansen, P., Peeters, D., Richard D., Thisse, J.F., (1985), The Minisum and Minimax Location Problems Revisited, Operations Research, 33, 1251-1265.

[9] Hansen, P., Jaumard, B., (1995), Handbook of Global Optimization, in Horst, R., Pardalos, P. (Eds), Kluwer Academic Publishers, Dordrecht. 
[10] Hansen, P., Jaumard, B., Tuy, H., (1995), Global Optimization in Location, in Z. Drezner (Ed.), 43-68, Facility Location: A Survey of Applications and Methods, Springer-Verlag, Berlin.

[11] Held, M., Wolfe, P. Crowder, H.P., (1974), Validation of Subgradient Optimization, Mathematical Programming, 6, 62-88.

[12] Hiriart-Urruty, J.B., Lemaréchal, C., (1993), Convex Analysis and Minimization Algorithms, vol 1, Springer-Verlag, Berlin.

[13] Horst, R., Tuy, H., (1993), Global Optimization (Deterministic Approaches), 2nd edition, Springer-Verlag, Berlin.

[14] Love, R.F., Morris, J.G., Wesolowsky, G.O., (1988), Facilities Location: Models and Methods, North Holland, New York.

[15] Minoux, M., (1986), Mathematical Programming: Theory and Algorithms, John Wiley and Sons, Chichester.

[16] Plastria, F., (1991), The Effects of Majority in Fermat- Weber Problems with Attraction and Repulsion in a Pseudometric Space, Yugoslav Journal of Operations Research, 1, 141-146.

[17] Plastria, F., (1992), GBSSS: The Generalized Big Square Small Square Method for Planar Single-Facility Location, European Journal of Operational Research, 62, 163-174.

[18] Plastria, F., (1995), Continuous Location Problems, in Z. Drezner (Ed.), 225-262, Facility Location: A Survey of Applications and Methods, SpringerVerlag, Berlin.

[19] Plastria, F., (1995), Continuous Location of Attracting and Undesirable Facilities, Semi-Plenary Papers Book, EURO XIV Conference, Jerusalem, 131-148.

[20] Rockafellar, R.T., (1970), Convex Analysis, Princeton University Press, Princeton, New Jersey.

[21] Romero-Morales, D., (1996), Optimización Global en Localización, Master Thesis, Universidad de Sevilla, Sevilla, Spain.

[22] Tuy, H., Al-Khayyal, F.A., Zhou, F., (1995), A D.C. Optimization Method for Single Facility Location Problems, Journal of Global Optimization, 7, 209-227. 